\title{
El destino de Sísifo. Régimen político y nueva Constitución en Cuba
}

\author{
Armando Chaguaceda \\ Profesor de El Colegio de Veracruz, Investigador de Gobierno \\ y Análisis Político AC, Xalapa, México \\ Email: xarchano@gmail.com \\ Eloy Viera Cañive \\ Fundación Colectivo+Voces, La Habana, Cuba \\ Email: eloyvierac@gmail.com
}

Recibido: 25.09.2020 | Aceptado: 02.12.2020

\begin{abstract}
Resumen: El texto aborda las transformaciones y persistencias político- institucionales, enmarcadas en el proceso (2016-2019) de debate y aprobación de una nueva Constitución en Cuba. Se ubica la experiencia dentro de la familia de los constitucionalismos autocráticos, específicamente en su modalidad soviética. Se analizan los elementos estructurales del régimen (post)totalitario vigente, poniéndolos en relación con las reformas e innovaciones legales aprobadas en la nueva Carta Magna. Al final, se evalúan las posibilidades que el nuevo marco constitucional ofrece para un mayor empoderamiento ciudadano, de cara a las demandas sociales y las transformaciones pendientes del modelo cubano.
\end{abstract}

Palabras Claves: Cuba; constitución; proceso constituyente; reformas; autocracia.

\section{The Fate of Sisyphus. Political Regime and New Constitution in Cuba.}

\begin{abstract}
The text addresses the political-institutional transformations and persistence, framed in the process (2016-2019) of debate and approval of a new Constitution in Cuba. The experience is located within the family of autocratic constitutionalism, specifically in its Soviet form. The current (post) totalitarian regime's structural elements are analyzed, based on the reforms and legal innovations approved in the new constitution. In the end, the article also evaluates the possibilities that the new constitutional framework offers for greater citizen empowerment analyzing social demands and pending transformations of the Cuban model.
\end{abstract}

Keywords: Cuba; Constitution; Constituent Process; Reforms; Autocracy.

\section{O destino de Sísifo. Regime político e nova Constituição em Cuba.}

Resumo: O texto aborda as transformações e persistências político-institucionais, enquadradas no processo (2016-2019) de debate e aprovação de uma nova Constituição em Cuba. A experiência está localizada na família dos constitucionalismos autocráticos, 
especificamente em sua forma soviética. São analisados os elementos estruturais do atual regime (pós) totalitário, colocando-os em relação às reformas e inovações jurídicas aprovadas na nova Carta Magna. Ao final, avaliam-se as possibilidades que o novo marco constitucional oferece para um maior empoderamento do cidadão, frente às demandas sociais e às transformações pendentes do modelo cubano.

Palavras-chave: Cuba; constituição; processo constituinte; reformas; autocracia.

\section{Como citar este artículo:}

Chaguaceda, A y Viera Cañive, E. (2021). El destino de Sísifo. Régimen político y nueva Constitución en Cuba. Polis Revista Latinoamericana, 20 (58), 58-77. doi: http://dx.doi. org/10.32735/S0718-6568/2021-N58-1578

A partir de 1959, la entonces joven dirección del triunfante proceso revolucionario concentró paulatinamente todo el poder político en sus escasas manos. El proyecto originariamente nacional-popular se convirtió en un Estado posrevolucionario, cuya dirección asumió el modelo soviético y construyó un régimen autocrático de signo post totalitario. Un régimen que se ha mantenido inmutable en sus elementos estructurales desde hace medio siglo. Ratificando la estabilidad y resiliencia de ese tipo de órdenes políticos (Lachapelle, 2020), en el mundo de la Post Guerra Fría.

Ese régimen ha tenido como envoltura y sostén legal un tipo de constitucionalismo afín a sus contenidos autocráticos (Chaguaceda, 2017). Peter Bertholz ha identificado, como rasgos fundamentales del constitucionalismo totalitario, la búsqueda y realización de valores supremos -que trascienden los valores y preferencias individuales autónomas y subordinan cualquier lealtad a otra persona, idea u organización-; la supresión de toda separación de poderes; la exclusividad del máximo liderazgo como fuente del derecho y autoridad para (re)interpretar, a su arbitrio, el verdadero sentido de la ley y el establecimiento de sanciones severas -en el Código penal y en otras normas, a veces no escritas- frente cualquier transgresión de aquella (Bertholtz, 1991). Esos rasgos han sido característicos del orden institucional vigente en la isla; en especial durante el largo período de dominio de Fidel Castro (1959-2006) en la política del país caribeño.

En lo relativo a la naturaleza del régimen vigente, este corresponde a la categoría de post totalitario. Bien sea en su modalidad carismática (Mujal y Saavedra, 1998) bajo el mando de Fidel Castro o en relación con el enfoque clásico de Linz y Stepan (1996) desarrollado por Morlino (2004) los elementos centrales del post totalitario están presentes en la isla tras la muerte del autócrata carismático. Liderazgo tendencialmente burocrático, movilización persistente pero limitada, menor peso de la ideología como mecanismo de cohesión y control social, aparición de mayor diversidad social sin reconocimiento del pluralismo político: todo eso caracteriza a la Cuba de los últimos años. La que, según la tabla siguiente, podemos ubicar en un post totalitarismo inicial con ciertos rasgos - ¿̇en tránsito? - a un modelo congelado. 
Tabla 1

Tipos y características de regímenes postotalitarios

\begin{tabular}{|c|c|l|l|c|}
\hline $\begin{array}{c}\text { Dimensiones de } \\
\text { Análisis }\end{array}$ & Inicial & Congelado & Maduro \\
\hline Pluralismo & Inexistente & Emergente & & Tolerado \\
\hline Ideología & Osificada & Vaciada & $\begin{array}{c}\text { Con elementos } \\
\text { tecnocráticos }\end{array}$ \\
\hline Movilización & $\begin{array}{c}\text { Espóradica, } \\
\text { ritualista }\end{array}$ & Abandonada & Perminada \\
\hline Liderazgo & $\begin{array}{c}\text { Burocrático } \\
\text { Colegial }\end{array}$ & $\begin{array}{c}\text { Burocrático, } \\
\text { Colegial }\end{array}$ & & Parta \\
\hline
\end{tabular}

Fuente: (Pasquino, 2011: 302)

\section{Los antecedentes de la Constitución actual}

En Cuba, las constituciones revolucionarias han cumplido con el fin de generar apariencias de sometimiento jurídico del poder e igualdad de derechos. Han sido promulgadas para servir de símbolos en un ambiente donde convertir la semiótica en un arma política es casi una ciencia.

El proceso de institucionalización de la "Revolución Cubana" culminó con la promulgación de la Constitución de 1976 y la posterior implementación de un sistema jurídico que reunía todas las características descritas por Bertholz. Incluso algunos de los más respetados catedráticos afines al gobierno cubano reconocen que la Carta Magna de 1976 no fue un "texto de límites al poder, sino de consagración de conquistas" (Prieto, Reflexiones en torno al carácter normativo de la constitución, 2005). La Constitución de 1976 fue más un símbolo que un instrumento jurídico con vocación de implementación. De acuerdo al criterio de Carlos Rafael Rodríguez,' la idea de dotar de institucionalidad al proceso iniciado en 1959 surgió en los dirigentes de la Revolución desde los primeros momentos, pero

"desde los balcones del palacio presidencial, muy poco después de iniciado el proceso de construcción revolucionaria, Fidel Castro habló de elecciones, y para sorpresa de todos los responsabilizados con esta revolución, un grito unánime surgió de aquella muchedumbre: ¡No!, ¡no!, ¡no!... desde entonces, la dirección de la revolución cubana se dio a la búsqueda de aquellas formas de gobierno que fueran más adecuadas a las características del proceso revolucionario" (Rodríguez, 1983).

Luego de casi dos décadas de búsqueda el gobierno revolucionario refrendó normativamente, a través de la Constitución de 1976, el poder del Partido Comunista como forma de gobierno. Las bases del texto habían sido establecidas previamente mediante tesis y

1 Carlos Rafael Rodríguez fue un comunista cubano Rodríguez con puestos de alta jerarquía dentro del gobierno revolucionario desde sus orígenes. Fue presidente del Instituto Nacional de Reforma Agraria (INRA, entre 1962 y 1965) y viceprimer ministro para asuntos exteriores (1972-1976). Incluso llegaría a ser conocido por algunos periodistas de la prensa extranjera como el "zar económico" de la Cuba revolucionaria. En la etapa de institucionalización constitucional de fue elegido y varias veces ratificado como vicepresidente del Consejo de Estado (1976-1993) y designado en el cargo de vicepresidente del Consejo de Ministros (1976-1997). Fue miembro del Buró Político del Partido Comunista de Cuba desde su refundación en 1965 y hasta poco antes de su muerte en 1997. 
resoluciones que sobre la Constitución y la ley de tránsito constitucional aprobara el Primer Congreso del Partido Comunista en diciembre de 1975 (Cuba P. C., 1975 a Habana). De ahí que no resultara rara la regulación del artículo 5 de la Carta Magna reconociendo al Partido Comunista como un organismo paraestatal encargado desde su superioridad, de dirigir al Estado y a la sociedad. La Carta Magna de 1976 fue obra de un órgano ad hoc subordinado a la dirección estatal y partidista, de la cual recibió indicaciones específicas en cuanto a contenidos y principios básicos (Prieto, Reflexiones en torno al carácter normativo de la constitución, 2005). Constituyó el símbolo del final del proceso de institucionalización de la Revolución Cubana. Un proceso que no fue diseñado por una Asamblea Constituyente como en 1901 cuando se construyó la institucionalidad de la naciente República cubana o como en 1940 cuando se reformó.

La Asamblea Constituyente era un mecanismo del que podía prescindir la Revolución, otras muchas constituciones se habían promulgado utilizando fórmulas diferentes. Sin embargo, de lo que, si no podía prescindir la Revolución, era de una Constitución en sí misma, pues había nacido para reivindicar la vulneración de una: la de 1940. Además, las constituciones como límites al poder del Estado y como base del ordenamiento jurídico son consustanciales a las concepciones modernas de democracia. En el mundo contemporáneo donde la comunidad internacional reconoce que la autodeterminación de un estado, también implica el establecimiento de "un gobierno que represente a todo el pueblo perteneciente al territorio sin distinción de raza, credo o color" (Unidas A. G., 1970), es difícil que algún estado o gobierno no intente como mínimo aparentar cumplir con esas expectativas.

Por eso, luego de 17 años del imperio de la Ley Fundamental dictada por el gobierno revolucionario provisional para fungir como norma marco durante la transición, fue promulgada la Constitución de 1976 que no tendría como misión organizar y limitar el poder político del Estado, sino exclusivamente legitimar un modelo totalitario cuyos límites no han descansado nunca ni en la Carta Magna ni en las leyes. La Carta Magna de 1976 -reformada en 1992 y 2002- significó el fin de la provisionalidad y la incardinación del Partido Comunista y de su monopolio político. Un proceso impulsado por comunistas cubanos pro soviéticos y del que fue protagonista la "generación histórica" que hizo la Revolución. La Constitución del 2019 es a su vez el símbolo del proceso de continuidad del monopolio político del Partido Comunista, pero esta vez impulsado por la generación histórica de la Revolución, y protagonizado por una generación nacida después de 1959.

Partiendo de esos antecedentes, el presente texto pretende abordar las transformaciones y persistencias político- institucionales de ese proceso de transferencia de poder producido entre los años 2018-2019, pero que tiene sus antecedentes en el momento en que Raúl Castro asumiera los roles de presidente de los Consejos de Estado y de Ministros en el año 2006. Así mismo, busca analizar los elementos estructurales del régimen (post)totalitario manifestados en la Carta Magna y en la legislación complementaria que hasta la fecha se ha promulgado. Se busca demostrar, a partir del análisis contextual en el que se promulga el texto, el proceso mediante el cual se construyó y los contenidos traducidos a éste, que la 
nueva Constitución no es un documento que pretenda realmente ajustar el sistema jurídico a la realidad imperante o servir como instrumento eficiente de la ciudadanía en su lucha por empoderarse e impulsar transformaciones políticas y sociales pendientes.

\section{Sucesión generacional autocrática y transferencia (limitada) del poder}

La Constitución del 2019 se desarrolló como colofón a un período de cambios que pudiesen ser considerados trascendentales para la Revolución Cubana. En el año 2013 Raúl Castro anunció que el mandato de cinco años que asumía en ese momento sería su último al frente del gobierno cubano. Los procesos naturales no perdonan ni a los políticos más influyentes, el ciclo de vida de la "generación histórica" estaba llegando a su fin, lo que quedó patentado con la muerte de su máximo líder tres años después en noviembre del 2016.

Asimismo, en diciembre del 2014, se anunciaba el inicio del proceso de normalización de las relaciones con el "enemigo histórico" de la Revolución Cubana: Ios Estados Unidos. Un proceso que se vio truncado en el 2016 con el ascenso a la presidencia de Donald Trump pero que tuvo un relevo con la firma del Acuerdo de Diálogo Político firmado entre Cuba y la Unión Europea (UE) ese propio año. Un Acuerdo que puso fin a más de dos décadas de "posición común" del bloque con relación a Cuba y que colocó al régimen cubano como una "democracia de partido único" (Europea, 2017) en el imaginario de la UE. En medio de esas confluencias, incluso habiéndose revertido el proceso de normalización impulsado por Barack Obama, una reforma constitucional, aparentemente abierta y deliberativa, era fundamental para demostrar la propensión del gobierno cubano a la apertura y a una renovación que no necesariamente implicaba alternancia. En octubre del 2017 el ministro cubano de Relaciones Exteriores Bruno Rodríguez Parrilla, resumió esa intención al anunciar en Washington nuevas medidas migratorias: "el gobierno de los Estados Unidos cierra y Cuba abre" (Rodríguez Parrilla, 2017).

La reforma constitucional parecía ser necesaria desde que Raúl asumió la presidencia interina de los Consejos de Estado y de Ministros en el año 2006 e impulsó un proceso de reformas económicas y de "fortalecimiento de la institucionalidad". A partir del 2007 el gobierno del menor de los Castro fue rearticulando la institucionalidad cubana que había sido bypasseada por Fidel Castro con la creación de organismos ad hoc con reales facultades ejecutivas como el Grupo de Apoyo² y la elevación de la Batalla de Ideas a institución, con una Vicepresidencia especial en el Consejo de Ministros y poderes ministeriales que le permitían invertir y administrar recursos millonarios en proyectos sociales de los que en su mayoría hoy solo queda el recuerdo.

El fortalecimiento de la institucionalidad no solo se convirtió en variable casi permanente en el discurso oficial de ese período (Castro, Cuba,cu, 2009), sino que se concretó con la transferencia de las oficinas administrativas de los diferentes programas de la Batalla de

2 El Grupo de Apoyo, fue un grupo creado por Fidel Castro para asistirlo en su dirección voluntarista de casi todas las áreas de la política nacional 
Ideas a los ministerios correspondientes según la rama y la purga que en el año 2009 removió a las principales figuras de la nueva generación, nacidas alrededor del año 1959 , que habían alcanzado posiciones importantes en los Consejos de Estado y de Ministros. Dicha purga evitó que se produjera un traspaso de poder en favor de las generaciones más jóvenes que sostenían la gestión de Fidel Castro. El saneamiento impulsado por Raúl no contribuyó a la transferencia de poder sino más bien a consolidar las estructuras de poder previas ratificando el hecho de que aquellos que participaron en la Revolución y los primeros años de gobierno siempre tendrían predominio sobre los más jóvenes. El cambio generacional que parecía venirse construyendo se desmontó y con esa acción se demostró la intención de la "generación histórica" de conservar el poder hasta que pudiesen encauzar una transferencia controlada a sectores generacionales más jóvenes que no conservaran la osadía de desconocer su influencia y capital político remanente.

Las reformas impulsadas por Raúl y sus proyecciones sistematizadas en los Lineamientos de la Política Económica del Partido y la Revolución aprobados en el VI Congreso del Partido Comunista de Cuba, planteaban en sí mismos un escenario incompatible con algunos de los más importantes postulados de la Constitución de 1976. Sin embargo, la idea de la promulgación de una nueva Constitución que sirviese de marco y límite a ese proceso nunca fue una premisa. Prácticamente el gobierno fue implementando normativamente los Lineamientos, de acuerdo a sus proyecciones y urgencias, sin reparar en la constitucionalidad.

Esa forma de ejercer el poder se incardina perfectamente en lo que Chofre-Sirvent (2020) ha considerado como una característica de la institucionalidad cubana: la desconsideración hacia lo jurídico, expresada no en la subordinación del poder a la Constitución, sino de la Constitución al poder. (Chofre-Sirvent, 2020). Lo cual, en otros textos (Chaguaceda, 2020) ha sido denominado cómo un enfoque inconfesadamente schmittiano de la relación entre política y legalidad, caracterizado por una subordinación de la soberanía popular al decisionismo autocrático. Signado por la coexistencia entre un Rule By Law formalizado en el diseño institucional y un Rule Despite Law operativo en mecanismos de control político.

Como ejemplos de esa característica de desconsideración hacia lo jurídico y subordinación de la ley al poder pueden citarse la promulgación en el año 2012 del Decreto-Ley 305 (Cuba C. d., Decreto-Ley 305. De las cooperativas no agropecuarias, 2012) que autorizaba la conformación de cooperativas no agropecuarias sin estar incluida esa forma de propiedad dentro del catálogo de numerus clausus utilizado por la Constitución de 1976 para regular las formas de propiedad válidas para el Estado Cubano. Así mismo se promulgó el 30 de diciembre del 2017 el Decreto-Ley 352 (Cuba C. d., Decreto-Ley 352. Sobre la adquisiciòn de la ciudadanía cubana por nacimiento de los nacidos en el extranjero de padre o madre cubanos, 2017) que permitía que los hijos de cubanos nacidos en el extranjero pudiesen adquirir la ciudadanía cubana contradiciendo el mandato constitucional que prohibía expresamente la doble ciudadanía. 
Otros muchos ejemplos, también podrían ser utilizados para ilustrar el hecho de que la constitucionalidad nunca ha sido un freno para las autoridades cubanas. En consecuencia, considerar a la Constitución del 2019 como un mero símbolo y no como un instrumento con real vocación y posibilidades de servir de límite a un estado totalitario, no debería resultar descabellado. Mucho menos si se evalúa que su promulgación coincide con un proceso de transferencia controlada de poder y con la necesidad de generar una imagen de apertura respaldada por uno de los actores más influyentes en la política internacional del momento: Ia Unión Europea. Con este criterio parece concordar también Chofre-Sirvent cuando afirma que: "la Constitución se ha utilizado, no para abordar profundos cambios en todos los órdenes de la sociedad, sino para relegitimar el sistema, fundamentalmente de cara al exterior" (Chofre-Sirvent, 2020).

No es casual que en el año 2013 al asumir por segundo período consecutivo el cargo de presidente de los Consejos de Estado y de Ministros, Raúl Castro haya declarado que su mayor satisfacción "sería la tranquilidad y serena confianza que sentía al ir entregando a las nuevas generaciones la responsabilidad de continuar construyendo el socialismo" (Castro, Cubadebate, 2013) y al mismo tiempo el Buró Político del Partido Comunista hubiese aprobado la creación de un Grupo de Trabajo para redactar una nueva Constitución (Acosta, Intervención al iniciar el debate del Proyecto de nueva Constitución en la Asamblea Nacional del Poder Popular., 2018). Tampoco es casual que la materialización formal de la entrega de poder se hubiese producido en abril del 2018 con la elección de Miguel Mario Diaz-Canel Bermúdez como presidente de los Consejos de Estado y de Ministros y en julio de ese mismo año se hubiese presentado el Proyecto de Nueva Constitución ante la Asamblea Nacional del Poder Popular para luego ser sometido a consulta popular.

En el discurso ofrecido durante la investidura de Diaz-Canel, Raúl Castro reconoció que su sucesor era el "único sobreviviente" de una "docena de jóvenes, la mayoría de los cuales llegó al Buró Político, pero no lograron materializar su preparación" (Castro, Trabajadores, 2018). En esa misma intervención anunció también que en la Constitución (refiriéndose a la que sería aprobada en febrero de 2019), no habría "cambios en el objetivo estratégico del trabajo del Partido..." y adelantó que uno de los objetivos que perseguían una vez que se aprobara la Carta Magna era unir nuevamente los cargos de Primer Secretario del Partido Comunista y de Presidente de los Consejos de Estados y de Ministros para que esa persona tuviera "en sus manos todo el poder y la influencia a ejercer".

Una señal clara de la intención del Partido Comunista de utilizar la Constitución para legitimar la transferencia de poder e instaurar a su vez un mecanismo que ofreciera seguridad al líder de esa fuerza política para mantener el control real, incluso habiendo perdido su condición de Jefe de Estado. Una idea que encuadra además con la perspectiva "raulista" de una entrega controlada y segura del poder que ofrezca siempre opciones de maniobra a los verdaderos detentores de éste: la cúpula del Partido Comunista. Así lo dejó claro el GeneralPresidente al afirmar que, en el 2021, fecha en que debe realizarse el VIII Congreso del 
único partido legal que existe en Cuba, Diaz-Canel deberá asumir también el rol de Primer Secretario. No obstante, dicha asunción la condicionó a "si trabaja bien, y así lo aprueban en el Comité Central" (Castro, Trabajadores, 2018).

En su intervención de despedida, Raúl Castro describió un proceso de supervisión paralelo que convierte los mecanismos de elección y control descritos en la Constitución del 2019 en meros postulados. Un proceso no normativizado pero diseñado para prevalecer incluso los mandatos del actual Presidente de la República. Pues según el Primer Secretario del Partido, ese es un mecanismo que debe repetir Díaz Canel con su sucesor "cuando al concluir sus períodos presidenciales, permanezca por otros tres años y hasta el Congreso al frente del Partido". De acuerdo al menor de los Castro ese sería un mecanismo indispensable "para viabilizar el tránsito seguro y ahorrar aprendizajes del sustituto" (Castro, Trabajadores, 2018).

\section{El proceso constituyente y la piedra de Sísifo}

Todas las proyecciones para constitucionales descritas por el General de Ejército, fueron solapadas en los debates impulsados durante el proceso de reforma constitucional iniciado oficialmente en junio del 2018. La participación popular en ese proceso fue calificada por el gobierno cubano como inigualable y sin precedentes en la historia del constitucionalismo moderno (Acosta, Intervención al iniciar el debate del Proyecto de nueva Constitución en la Asamblea Nacional del Poder Popular., 2018). Las cifras se utilizaron para demostrar el apoyo del pueblo a los postulados constitucionales; con independencia de los rejuegos de poder que iban más allá del texto ofrecido a la ciudadanía.

Una lectura fría de las cifras ofrecidas por el propio gobierno cubano sobre los resultados de la consulta pudiese llevar a concluir que ciertamente el proceso contó con un mayoritario respaldo popular. Sin embargo, detrás de esos números se esconden elementos que hacen dudar de la validez de la metodología empleada para el cómputo y por ende de sus resultados. Las cifras oficiales indican que durante la consulta se realizaron 133 mil 681 reuniones a las que asistieron 8 millones 945 mil 521 personas (Acosta, Cubadebate, 2018). Dicha cifra equivale aproximadamente al $78 \%$ de la población cubana y excede en casi 240 mil personas la cifra oficial del padrón electoral con el que contaba Cuba al momento del referendo (Cubadebate, Cubadebate, 2019).

Las reuniones se desarrollaron en los centros laborales, de estudio y en los barrios. Sin embargo, no existía ninguna prohibición o mecanismo para controlar cuando la misma persona participaba en más de una reunión, lo que pudo haber dado lugar a duplicidades en los conteos y puede explicar el exceso existente entre la cifra de participantes en las reuniones y el padrón electoral. También resulta importante incluir como variable de análisis de los datos, sobre todo en un ambiente post totalitario como el cubano, la influencia que pudieron haber ejercido en la participación las dinámicas de control que se reproducen al interior de los ambientes laborales y estudiantiles, donde se produjeron casi el $40 \%$ de las reuniones. En 
ese sentido, 45452 reuniones se produjeron en centros laborales controlados por el Estado Cubano y 4841 se produjeron en los centros universitarios y de enseñanza media del país también bajo influencia estatal.

Analistas foráneos que han estudiado el proceso de consulta popular han considerado que con independencia de los números ofrecidos, "los procesos deliberativos implementados en contextos no democráticos como el cubano tienden a no cumplir con los requisitos mínimos para ser considerados abiertos y plurales" (Welp, 2020). Resaltan la idea de que a pesar de que las cifras pueden mostrar una participación masiva, la "oposición política está prohibida y es perseguida" (Welp, 2020), lo que genera limitaciones no despreciables en la calidad y real influencia de los espacios deliberativos.

Por otro lado, también se señala como un elemento cuestionable la forma en la que se procesaron los datos y la decisión sobre su inclusión en el texto definitivo. Las propuestas de la ciudadanía fueron recopiladas por un Equipo Nacional de Procesamiento, que utilizó un software desarrollado por la Universidad de Ciencias Informáticas, un centro de desarrollo emblemático del gobierno, fundado por Fidel Castro y señalado por el posible mantenimiento en su seno de granjas de troles y controladores del ciberespacio nacional (Jimenez Enoa, 2017). La fuente primaria de esas informaciones, o sea las actas de las reuniones donde se compilaron las intervenciones de cada una de las reuniones no se hicieron públicas y por ende no fue posible contrastar los resultados ofrecidos. La información compilada por el Equipo de Procesamiento fue evaluada con posterioridad por un Grupo de Análisis compuesto por ocho miembros de la Comisión Redactora de la Constitución y 22 expertos de diferentes ramas del derecho de los que nunca se conoció las formas y los criterios que fueron utilizados para su selección. Ese Grupo de Análisis fue el encargado de evaluar las propuestas y proponer a los redactores los cambios que consideraron pertinentes para el primer borrador sometido a consulta popular. Este mecanismo, llevó a Welp a considerar a la consulta popular desarrollada en Cuba "como un proceso de participación con incidencia controlada" (Welp, 2020).

Sin embargo, sería pertinente acotar que la participación ciudadana fue solo uno más de los elementos controlados dentro de todo el proceso de reforma constitucional. Hay razones bastante evidentes para pensar que desde su origen fue un proceso manejado por el Partido Comunista. Desde el 2013, la propia organización política había iniciado de forma no publicitada (Toque, YouTube, 2018), la redacción de un borrador de Carta Magna que es muy presumible haya sido ofrecido como base a la Comisión que se denominó Redactora y de la que formaron parte al menos 7 personas que habrían pertenecido al Grupo de Trabajo inicial conformado cinco años antes (Acosta, Intervención al iniciar el debate del Proyecto de nueva Constitución en la Asamblea Nacional del Poder Popular., 2018). La Comisión Redactora estuvo compuesta por 33 diputados, de los cuales solamente dos no eran militantes del Partido Comunista y el $27 \%$ eran miembros del Buró Político o el Comité 
Central de la organización partidista. La Comisión no sesionó permanentemente, ni siquiera antes de presentar el borrador a la Asamblea Nacional y a pesar de la brevedad del tiempo con el que contaron para esa labor. ${ }^{3}$

El gobierno cubano ha sido capaz, en los marcos del régimen vigente, de mutilar la iniciativa política de la ciudadanía y la operatividad de las instituciones. Sin embargo, la semiótica ha sido siempre parte integrante de su arsenal político y apelando a ella han generado espacios y herramientas que han servido para articular toda una teoría sobre el carácter directo y popular de la democracia cubana, A pesar de eso, un análisis del fondo y la forma en que se expresa dejan muestras suficientes para asegurar que en su diseño subyace una marcada intención de limitar la influencia y participación ciudadana en el poder político. Ese análisis cabría aplicarlo también al proceso de consulta popular generado en torno a la Constitución del 2019. Los números le permitieron al gobierno afirmar "sin vanidad...que era un ejercicio único de democracia real y efectiva y de un proceso constituyente igualmente paradigmático con el pueblo como protagonista verdadero" (Acosta, Cubadebate, 2018). Sin embargo, esa es una afirmación más que discutible y contrastable.

A la larga y a pesar del "protagonismo del pueblo", su incidencia estuvo tan controlada que el gobierno logró desligarse selectivamente de algunos de los más trascendentales deseos populares. Fue así como los reclamos de más de 11 mil cubanos que solicitaron la elección directa del Presidente de la República no fueron escuchados, mientras que si lo fueron otros 575 (Cubadebate, Cubadebate, 2018) que solicitaron fuera retomado, al menos como aspiración política, el término comunismo desterrado de la primera versión del Proyecto de Constitución. La consulta popular más que un mecanismo para ofrecer opciones de participación a la ciudadanía fue utilizado para evaluar los niveles de consenso con relación a algunos temas incluidos en el primer borrador del texto constitucional. Resultaba complejo que una ciudadanía sometida mayoritariamente a la influencia de una única matriz de opinión, sostenida por el sistema estatal de propaganda y dirigida a resaltar las bondades del Proyecto, pudiese aportar alternativas a las opciones que desde arriba se le ofrecían. De ahí que las atenciones se concentran en temas socialmente sensibles como el matrimonio igualitario o de fácil comprensión política como la elección directa del Presidente de la República.

La consulta popular fue otra muestra de que la ciudadanía cubana sufre la maldición de Sísifo. Debe arrastrar cuesta arriba sus esperanzas de ser escuchada por un Estado que siempre termina condenándola a repetir infructuosamente la tarea. La consulta popular pretendía allanar un resultado favorable al gobierno en el referéndum. Nunca fue diseñada para convertirse en un mecanismo de incidencia efectivo sino en un medio para verificar las múltiples sospechas que el gobierno mantiene respecto a su ciudadanía. Las que fueron, en parte, corroboradas -a pesar de lo dicho por la propaganda- con los resultados del referéndum.

3 La Comisión fue aprobada el primero de junio del 2018 por el Consejo de Estado y el Proyecto se presentó a la Asamblea Nacional el 21 de julio de ese propio año. 


\section{El referéndum}

En el referéndum de aprobación de la Constitución del 2019 participó aproximadamente el $90 \%$ de los votantes inscritos en el padrón electoral. Una cifra que se encuentra en consonancia con las de participación en la consulta y que conjuntamente con el $86,85 \%$ de aprobación, fue utilizada por el gobierno para ofrecer una imagen de respaldo mayoritario a la Constitución y con ello a la continuidad del régimen político legitimado por ésta. Como todas las cifras relacionadas con el proceso de reforma, los resultados del referéndum también hay que evaluarlos sobre la lógica del valor que el gobierno cubano le confiere a la semiótica como instrumento para generar realidades políticas inconsistentes. Las lógicas de la accountability vertical electoral no pueden ser aplicadas al modelo cubano. El régimen cubano no se sostiene en el poder sobre la base de la legitimidad que le confiere el voto popular. Escasamente se ha sometido a procesos eleccionarios donde el voto define la permanencia de sus más altos dirigentes o condiciona decisiones gubernamentales.

Ante la ausencia de soluciones legales diferentes al referendo para aprobar una nueva Constitución y, el elevado costo político que pudiese tener en la sociedad cubana contemporánea aprobarla sin el respaldo del voto directo de la ciudadanía, el gobierno cubano, a pesar de que ha rehuido históricamente los procesos plebiscitarios y referendarios, no pudo abstraerse en esta oportunidad de un proceso eleccionario contencioso. Una solución de la que nunca ha sido partidario pues ha insistido en el hecho de que, atendiendo a las características no competitivas de su modelo electoral, el apoyo a su gestión habrá que buscarlo en la participación masiva en las elecciones (Exteriores, Ministerio de Relaciones Exteriores, 2018). Una participación que no está exenta de presiones y que está concebida como un criterio de señalamiento y posterior discriminación política.

De ahí que los resultados del referéndum, haya que leerlos también desde un prisma diferente al de victoria absoluta tal cual lo hiciera el gobierno. Ante la ausencia de ejemplos, más allá del acontecido en 1976, que puedan utilizarse para comparar los resultados del referéndum, no resulta desatinado afirmar que el respaldo del 78,3\% del padrón electoral que recibió la Constitución del 2019 y con ella el régimen cubano, supone el menor nivel de apoyo al gobierno en unas elecciones en la Cuba postrevolucionaria. Lo que sumado al $10 \%$ de abstención, el 8,1\% de negativa de respaldo y el 4,1\% de anulación (Cubadebate, Cubadebate, 2019), demuestra que el consenso popular de apoyo al gobierno cubano existente en 1976 y demostrado con un $99,1 \%$ de participación y un $98 \%$ de aprobación, no se corresponde en lo absoluto con la realidad cubana actual. En total, cerca del $22 \%$ del padrón (aproximadamente 1 millón 915 mil electores) no apoyaron de una forma $u$ otra el proyecto (ya sea no yendo a votar, votando en contra, anulando su boleta o dejándola en blanco).

A pesar de ello, el gobierno cubano continúa actuando como en los años 70: bloqueando la participación política a todos aquellos que cada vez más visiblemente se le oponen. Una situación que contrasta mucho más si se adicionan a todas esas variables la posible 
influencia que pudo haber tenido en el referéndum el voto de la comunidad cubana en el exterior. El gobierno, como muestra de concordia, invitó a todos los cubanos de la diáspora a participar en la consulta popular y para ello habilitó un sitio web donde se computarían sus criterios y propuestas (Exteriores, Nación y Emigración, 2018). Sin embargo, después les negó injustificadamente a muchos, su participación en el referendo desde sus lugares de residencia.

La ley Electoral vigente al momento de producirse el referendo constitucional reconocía la obligación del Estado Cubano de garantizar que los electores que se encontraban fuera del territorio nacional en el momento de un referendo, pudiesen ejercer su derecho al voto (Popular, Ley Electoral, 1992). La condición de elector en aquel momento solamente estaba condicionada a la residencia legal, la cual mantenían muchos cubanos que a pesar de residir efectivamente en territorio extranjero ingresaban cada 24 meses a la isla. Empero, la obligación del Estado fue incumplida y no por cuestiones infraestructurales pues el Ministerio de Relaciones Exteriores, creó las condiciones necesarias para que las personas que se encontraban de misión oficial en el extranjero pudiesen participar en el referendo (EFE, 2019).

El gobierno cubano evitó así, que ciudadanos no sometidos a las mismas presiones y controles que los residentes en el archipiélago participaran en el referéndum. El monopolio de la vida política y jurídica de la nación cubana, que detenta el Partido Comunista, le permitió no sólo vulnerar con facilidad y sin consecuencias su propia ley y evitar así el voto de un sector mayoritariamente crítico con la gestión del gobierno. Sino también ajustar sin resistencias los espacios que en el ordenamiento jurídico había dejado y que pudieran convertirse en una herramienta de la ciudadanía para disputar poder legítimamente y por ende constituir una amenaza a su control hegemónico.

En septiembre del 2019, por unanimidad y sin criterios encontrados, la Asamblea Nacional del Poder Popular aprobó una nueva Ley Electoral (Popular, Ley 127: Electoral, 2019). Con ella el gobierno cubano limitó aún más las posibilidades de la diáspora cubana de participar en la vida política del Estado al reconocer a la "residencia efectiva" como condicionante para el ejercicio de los derechos políticos en Cuba. La norma condicionó el derecho al voto a la presencia física en el territorio nacional y a la manifestación de actos que evidenciaran la voluntad del elector de mantener a Cuba como domicilio permanente (Cilano Pelaez, s.f.). Así, se eliminó de un plumazo la causa de que meses antes se le interpelara y se demostrara sin respuesta, sus rasgos autocráticos.

Con la Ley Electoral derivada de la nueva Carta Magna se cerró también la puerta a la implementación de un voto cubano en el extranjero y se posibilitó la exclusión de ese derecho ante el criterio selectivo de la mesa electoral. Al mismo tiempo se bloqueó por completo la posibilidad de que esa comunidad con posibilidades de articulación, inexistentes al interior de la Isla, participara en próximos referendos como el anunciado para la aprobación del futuro Código de Familia. 
Un referendo convocado desde las propias disposiciones de la Constitución del 2019 y que es el resultado del proceso de negociaciones y evaluación de fuerzas derivado de la consulta popular. Durante los debates del Proyecto de Constitución, las fuerzas conservadoras -nucleadas alrededor de ciertas Iglesias y comunidades religiosas- demostraron número y decisión de boicotear el referéndum, lo que constituía una amenaza clarísima al mito de la unidad y el apoyo popular en torno al Partido y al Gobierno. Ante el riesgo de sufrir un menoscabo de ese símbolo, el régimen cubano cedió con la principal exigencia de esos sectores y terminó modificando la propuesta original que reconocía expresamente el matrimonio igualitario (Service, 2018).

Luego de la consulta popular, el texto si bien no cerró completamente la puerta a ese derecho de la comunidad LGBTIQ, si condicionó su reconocimiento posterior en el Código de Familia a la celebración de otro referéndum de esencia plebiscitaria en el año 2021 (Popular, Constitución de la República de Cuba, 2019). El gobierno demostró así, su propensión a mantener la semiótica alrededor de su legitimidad antes de cumplir con una de sus obligaciones: garantizar de forma irrestricta y sin condicionamientos los derechos humanos de las "minorías".

\section{Más allá de la consulta y el referéndum: la sala de máquinas permanece intacta.}

De forma general el proceso de consulta popular, los resultados del referendo y la expansión nominal de algunos intereses y derechos de la ciudadanía en la Nueva Constitución cubana, ha servido como una especie de cortina de humo para mantener intacta la sala de máquinas de su predecesora. La metáfora de Roberto Gargarella al referirse a uno de los principales señalamientos del constitucionalismo latinoamericano (Gargarella, 2014) es perfectamente aplicable a lo sucedido en el proceso de reforma constitucional cubano.

La Carta Magna del 2019, utilizó por primera vez el término derechos humanos que había sido rechazado durante mucho tiempo por el régimen al estar estrechamente vinculado a la forma en la que la ciudadanía percibía y nombraba a la oposición tradicional. Incluyó nuevos derechos, reguló conceptos internacionales como debido proceso y modeló incluso garantías judiciales para el ejercicio de algunos de los derechos. Sin embargo, no introdujo cambios importantes en la organización del poder, sino que más bien terminó convirtiéndose en un instrumento para normativizar una forma de gobierno personalista que había caracterizado desde siempre a la Revolución Cubana pero que se había intentado ocultar bajo la retórica de que las decisiones estatales colegiadas estaban en la base de la democracia socialista cubana. Idea sostenida con la existencia de órganos de estado y de gobierno colegiados, cuya presidencia estuvo compartida siempre por la misma persona, pero teóricamente controlada por las decisiones de la mayoría de los miembros de los Consejos de Estado y de Ministros. 
Para conservar el núcleo real del poder, la Carta Magna mantuvo intacta la cláusula de intangibilidad introducida en el año 2002 a la Constitución de 1976 y que está dirigida no al blindaje de derechos, sino a garantizar la perpetuidad del "sistema socialista" indisolublemente ligado al monopolio político del Partido Comunista. Instauró también la figura del Presidente de la República, un órgano unipersonal inexistente en Cuba bajo la vigencia de la Constitución de 1976 y le reconoció no solo como Jefe de Estado, sino que también le confirió funciones de dirección del Gobierno con independencia de la existencia de un Primer Ministro. De esa forma reflejó el ideal de concentración de poder definido como objetivo estratégico por el Partido Comunista (Castro, Trabajadores, 2018). Un poder que será indiscutido porque con independencia del equilibrio aparentemente establecido sobre la base de considerar a la Asamblea Nacional del Poder Popular como el órgano supremo del Estado Cubano y reconocerle la potestad de revocar el mandato del Presidente de la República, la Carta Magna mantuvo inalterada algunas de las bases que convierten al parlamento cubano en un órgano prácticamente inoperante.

La Constitución del 2019 mantuvo el carácter no permanente de la Asamblea Nacional y sus exclusivos dos períodos de sesiones anuales, situación que facilita su existencia como el sucedáneo falaz de un Parlamento que sigue abdicando de sus funciones deliberante, legislativa, contralora y de custodia/reformas constitucionales, a favor de los Consejos de Estado y Ministros y ahora también del Presidente de la República. Limitada además por la falta de profesionalización de sus miembros, sus integrantes, a pesar de sus atribuciones legales, continúan careciendo de autonomía y no muestran iniciativa más allá de puntuales temas -casi siempre derivados de la administración y ejecución puntual de ciertas políticas- y encomiendas desde arriba. Muestra de ese particular es que, en más de 40 años de institucionalización, ni un solo diputado ha utilizado su iniciativa legislativa para presentar un Proyecto de Ley y solicitar su debate ante el pleno de la Asamblea Nacional.

La profesionalización de la labor de los representantes del Poder Popular, incluidos los diputados es indispensable para el ejercicio de esa autonomía. Por esa razón es una vieja añoranza de un sector (Orrio, 2013) de la intelectualidad de izquierda que cree en la perfectibilidad del modelo cubano. La idea retomó fuerza a la sazón del debate generado durante la consulta popular, sin embargo, no tuvo traducción a la nueva Carta Magna y recibió, al igual que la comunidad cubana residente en el exterior, un golpe mortal con la aprobación en octubre del 2019 de la nueva Ley Electoral. La norma jurídica redujo el tamaño del Parlamento y con ello la representación de los delegados barriales, únicos dentro de todo el entramado estatal, directamente propuestos y elegidos por la ciudadanía.

La falta de autonomía de los diputados y de la dirección de la Asamblea Nacional ha quedado evidenciada durante el año 2020. En todo el año, sin haberse emitido ni tan siquiera un comunicado justificativo, la Asamblea Nacional no sesionó hasta el mes de octubre. La inactividad de la Asamblea no sólo no se justifica legalmente, sino que ha dilatado el Cronograma Legislativo (Toque, El Toque, 2019) aprobado por ella misma para complementar la Constitución. 
El mentado Cronograma comprendía la aprobación en el mes de julio de una Ley de Revocación de Mandato que no fue aprobada hasta el mes de octubre. Una Ley que terminó regulando un mecanismo ineficiente que no encuadra dentro de los estándares de la revocación clásica y excluye la participación del electorado, pero que fue aprobada por unanimidad en el seno de la Asamblea y sin haberse producido un comentario de parte de ninguno de los diputados durante la sesión en la que teóricamente debió haberse discutido. La inactividad y pasividad de la Asamblea Nacional y sus miembros y la dilación del Cronograma Legislativo, pone también sobre la mesa el fantasma de la inconstitucionalidad por omisión que sufrió la Constitución de 1976 en algunas materias como los derechos de reunión, manifestación y de libertad de prensa.

Paralelamente al receso injustificado de la Asamblea Nacional, el gobierno, amparado en la crisis generada por la COVID 19, ha hecho uso de poderes excepcionales que le sobran, para limitar aún más, algunos derechos fundamentales. El accionar del Gobierno durante la crisis y la inoperancia de la Asamblea Nacional del Poder Popular certifican las dudas, mostradas también durante el proceso de consulta popular, sobre la eficiencia de los mecanismos de control constitucional implementados por la nueva Carta Magna, entre ellos las garantías judiciales de derechos.

La Constitución de 2019, aunque de forma limitada, introdujo a diferencia de su predecesora, la garantía judicial para la defensa de algunos derechos constitucionales. Las materias constitucionales habían sido, hasta su incorporación en la Constitución vigente, expresamente excluidas del conocimiento de los tribunales. Sin embargo, y como muestra de la esencia totalitaria que no puede ocultar el régimen cubano, la propia Constitución estableció una reserva de esa garantía sólo para aquellos derechos que sean dispuestos en una normativa posterior, que determinará no solo los objetos de protección sino también las formas de acceder a los tribunales para discutir ese tipo de conflictos.

La garantía judicial condicionada es un atentado contra la naturaleza viva de la Constitución y su capacidad de convertirse en norma de aplicación directa. Una aspiración de un sector importante de la academia cubana y de la ciudadanía que ha visto fenecer la posibilidad de apelar a la Constitución como garante de derechos y límite a los poderes absolutos del Estado.

La pérdida de valor jurídico de la Constitución es un problema originado desde los primeros años de la Revolución Cubana y muestra de los resortes que mueven al poder en Cuba más allá del imperio de la ley. Desde 1960 el Dr. Fernando Álvarez Tabío en su obra el "Recurso de inconstitucionalidad", se preocupaba por el valor normativo de la Constitución al destacar "que el supremo órgano de justicia no podía apartarse de sus normas ni tolerar una ley ordinaria que ofendiera los derechos esenciales consagrados en ella". Los análisis de Álvarez Tabío se producían motivados por los pronunciamientos del Tribunal Supremo Cubano, que en 
ese mismo período asumió posiciones completamente contrarias con respecto a la Constitución, al limitar su valor jurídico como ley aplicable o norma de contraste (Prieto, En pos de la aplicabilidad directa de la constitución cubana de 1976 (un breve comentario), 2008).

Debe recordarse que la Sala de Garantías Constitucionales, en algunas sentencias de esos años, se opuso a la aplicación directa de la Constitución respecto a algunos preceptos y reclamó la espera de leyes de desarrollo que instrumentarían y permitirían aplicar los contenidos de la Ley Fundamental. De acuerdo al criterio de la profesora Martha Prieto, varios fueron los elementos que condicionaron el desarrollo de estas posturas judiciales. Fundamentalmente "evitar que se judicializar la controversia política". Una postura que cumplía con el deseo "revolucionario" de limitar la posibilidad de la clase política yeconómica desplazada por la Revolución de ampararse en preceptos que se conservaron en la Ley Fundamental de 1959 de forma idéntica a como estaban expresado en la Constitución de 1940 y solicitaran "una interpretación constitucional con el espíritu de la vieja ley, lo cual implicaría un desconocimiento de las nuevas condiciones sociopolíticas y económicas en que vivía el país" (Prieto, En pos de la aplicabilidad directa de la constitución cubana de 1976 (un breve comentario), 2008). Pero también con ello se fue sentando una práctica desde el propio Tribunal Supremo de aplicar la ley y no la Constitución, bajo la justificación de la defensa de la "construcción de la nueva sociedad".

La disminución del valor de la Constitución como norma jurídica de aplicación directa, alcanzó niveles superiores luego de la entrada en vigor de la Constitución de 1976. El texto dotó a la Asamblea Nacional de facultad constituyente y le confirió el control de la constitucionalidad de las normas jurídicas. Ese proceso terminó convirtiendo a la Constitución en un texto político aspiracional con la misma falta de vitalidad que el encargado de su defensa. Este modelo de control de la constitucionalidad favorable al ejercicio arbitrario de los poderes omnímodos del Estado Cubano por su demostrada inefectividad ha sido mantenido también como un paradigma por la Constitución de 2019. Esta reproducción convierte a su vez en una quimera lo que pudo haberse evaluado como un avance para la participación política de la ciudadanía derivado del proceso de reforma constitucional. La Ley de Organización y Funcionamiento de la Asamblea Nacional del Poder Popular y el Consejo de Estado (Popular, Ley 131: de la Organización y Funcionamiento de la Asamblea Nacional del Poder Popular y el Consejo de Estado, 2020), complementaria de la Constitución, reguló un recurso de inconstitucionalidad popular que con anterioridad no existía en el ordenamiento jurídico cubano. Permite que 500 ciudadanos puedan presentar ante la Asamblea Nacional una solicitud para declarar inconstitucional una norma jurídica determinada. Sin embargo, esa facultad ciudadana puede resultar completamente ineficiente cuando se trata de normas de jerarquía superior como las Leyes y los DecretosLeyes. Las primeras difícilmente serán revocadas por sus mismos impulsores y las segundas requieren ser ratificadas o revocadas por la propia Asamblea Nacional luego de ser emitidas (Popular, Constitución de la República de Cuba, 2019). Un Decreto-Ley que ha 
sido ratificado expresamente o por omisión como también es costumbre en la práctica del Parlamento cubano, difícilmente será revocado por los mismos que lo han ratificado, con independencia del cúmulo de firmas y argumentos que sostengan la petición.

Las deficiencias en el control de la constitucionalidad permiten además que la Carta Magna se convierta en una norma vitrina que pueda ser modificada y limitada sin consecuencias por normas inferiores. La promulgación del Decreto-Ley 370 (Estado, (Cuba C. d., Decreto-Ley 370, sobre la Informatización de la Sociedad en Cuba, 2019) es una muestra de esos riesgos y de la esencia post totalitaria del régimen cubano.

El ejercicio de la libertad de expresión estuvo siempre condicionado por la Constitución de 1976 a los "fines de la sociedad socialista". En la Constitución del 2019, ha sido reconocido llanamente y sin condicionamientos. No obstante, ha sido limitado impunemente en una norma emitida por el Consejo de Estado: el Decreto-Ley 370. En julio del 2019 a escasos meses de entrar vigor la nueva Constitución, la Gaceta Oficial de la República publicó el polémico Decreto-Ley, que utiliza categorías como la moral, las buenas costumbres y el interés social, para limitar el derecho a la libertad de expresión, cuyo reconocimiento sin condicionantes parecía ser una conquista del proceso de reforma constitucional. El Decreto hasta la fecha no ha sido ni ratificado ni revocado por la Asamblea Nacional del Poder Popular; empero, esa situación no ha limitado su vigencia. Desde su promulgación se ha empleado no sólo para controlar a periodistas independientes y agentes de influencia opuestos al régimen cubano, sino también contra ciudadanos comunes que utilizan las redes sociales y el Internet como medios para expresar inquietudes (Cuba y su Decreto-Ley 370: Aniquilando la libertad de expresión en Internet.).

Dicha norma reconoce como contravención el hecho de "difundir, a través de las redes públicas de transmisión de datos, información contraria al interés social, la moral, las buenas costumbres y la integridad de las personas" y establece multas de hasta 3000 pesos moneda nacional (aproximadamente 120 dólares) y el decomiso de los equipos utilizados para las publicaciones. Ese amparo legal se ha utilizado para sancionar, de acuerdo a reportes de prensa (Padrón Cuento, 2020), a más de una veintena de activistas, periodistas independientes y ciudadanos comunes entre enero y mayo del 2020. Lo dispuestos por el Decreto-Ley 370 contraviene los estándares internacionales en materia de libertad de expresión y restringe ese derecho en base a objetivos que no son legítimos según lo previsto en el Derecho Internacional de los Derechos Humanos. "La nueva Constitución cubana, el Decreto ley 370, y el accionar del Estado cubano, contravienen plenamente el artículo 19 de la Declaración de los Derechos Humanos, y el artículo 19 del Pacto Internacional de Derechos Civiles y Políticos, firmado por Cuba el 28 de febrero de 2008; pero que lleva largos años sin ratificar. Este marco, bajo el cual el Estado cubano puede sancionar el uso de las Tecnologías de la Información y Comunicación, genera un efecto inhibitorio de la libertad de expresión ejercida a través de estas herramientas y plataformas, en tanto pesa una amenaza real y permanente de sanción para las personas, prácticamente para cualquier 
discurso que emitan y que discrecionalmente los órganos del Estado pueden calificar como una contravención legal, y posteriormente conducir a la privación de libertad" (Cuba y su Decreto-Ley 370: Aniquilando la libertad de expresión en Internet.).

\section{Conclusiones}

"Esta Constitución va a organizar el diagrama estatal del país. Pero hay una fuerza que está por encima del Estado, que es dirigente y superior: es el Partido. Luego, la Constitución no puede trazarle directrices al Partido"

José Luis Toledo Santander, Presidente de la Comisión de Asuntos Constitucionales y Jurídicos, Asamblea Nacional4

La aprobación de la Constitución de 2019 no ha variado la esencia post totalitaria del régimen político cubano. No habilita canales que realmente permitan a la ciudadanía utilizar sus normas e instituciones para participar autónomamente y limitar los poderes de un Estado autocrático. ${ }^{5}$ La nueva Constitución ha sido más bien el símbolo pensado para legitimar a una nueva generación de líderes.

Con independencia del nuevo modelo de Estado que se anuncia, los resortes reales del poder no se mueven. Constituyen la principal barrera para cualquier forma de democracia, de socialismo y de expresión de la diversidad natural de personas e intereses que conforman la nación cubana.

\section{Bibliografía}

Acosta, H. (12 de diciembre de 2018). Cubadebate. Recuperado de Cubadebate: http://www.cubadebate.cu/noticias/2018/12/18/presentan-cambios-en-el-proyecto-de-constitucion-derivados-de-la-consulta-popular/\#. X22RUIVKjIV

Acosta, H. (22 de diciembre de 2018). Intervención al iniciar el debate del Proyecto de nueva Constitución en la Asamblea Nacional del Poder Popular. La Habana, Cuba.

Bertholtz, P. (1991). The Constitution of Totalitarianism. Journal of Institutional and Theoretical Economics, 425-440.

Castro, R. (22 de diciembre de 2009). Cuba,cu. Recuperado de Cuba.cu: http:// wWw.cuba.cu/gobierno/rauldiscursos/index2.html

Castro, R. (24 de febrero de 2013). Cubadebate. Recuperado de Cubadebate: http://www.cubadebate.cu/especiales/2013/02/24/raul-castro-la-mayor-satisfaccion-es-la-tranquilidad-y-serena-confianza-que-sentimos-al-ir-entregan-

4 Ver https://www.youtube.com/watch? V=5Fgh_IMKWhA

5 Según Freedom House, Cuba es el país con menor libertad en la red de las Américas y el cuarto peor a nivel mundial (Shahbaz, 2019), entre 65 monitorizados. Conforme el Índice CIVICUS Monitor de la vigencia de las libertades de asociación, expresión y reunión pacífica, su espacio cívico es calificado como "cerrado" (CIVICUS, 2019). El Relator Especial sobre la libertad de opinión y de expresión de Naciones Unidas mostró su preocupación por los diversos mecanismos de represión vigentes (Unidas R. E., 2019). La Relatoría de Libertad de Expresión del sistema interamericano (Humanos, Informe Anual, 2019) reseñó la persecución sistemática a periodistas independientes que difunden información y opiniones sobre temas de interés público y en su comunicado del 18 de abril de 2020 (Humanos, Comunicado de Prensa R78/20, 2020), expresó su preocupación por las restricciones a la libertad de expresión y el acceso a la información en la respuesta del Estado a la COVID 19, resaltando los casos de periodistas multados en virtud del Decreto-Ley 370. 
do-a-las-nuevas-generaciones-la-responsabilidad-de-continuar-construyendo-el-socialismo-fotos/\#.X2YfpWhKhyw

Castro, R. (2018 de abril de 2018). Trabajadores. Recuperado de Trabajadores: http://www.trabajadores.cu/20180420/el-partido-comunista-apoyara-y-respaldara-resueltamente-al-nuevo-presidente-video/

Chaguaceda, A. (2020) Schmittianos del Caribe, Rialta, (45), Recuperado de: https://rialta.org/schmittianos-del-caribe/

Chaguaceda, A. (. (2017). Constitucionalismo autocrático en Cuba: mito y realidad. En B. C. Rojas. R., El cambio constitucional en Cuba. Madrid, España: Fondo de Cultura Económica.

Chofre-Sirvent, J. (2020). La Constitución cubana del 2019 y el constitucionalismo socialista: realidades y desafíos. FORO EUROPA CUBA.

Cilano Pelaez, J. (s.f.). DemoAmlat. Recuperado de DemoAmlat: https://www. demoamlat.com/constitucion-y-ley-electoral-en-cuba-vino-nuevo-en-odresviejos/

CIVICUS. (2019). People Power Under Attack. Recuperado de https://monitor.civicus.org/PeoplePowerUnderAttack2019/? fbclid=IWAR 1 mkw34fFcsOsMMMROKJC_MLy9pCyJEb809ChD-p8Q6ciJ0jfAtRnpBLZg

(s.f.). Cüba y su Decreto-Ley 370: Aniquilando la libertad de expresión en Internet. Recuperado de https://www.cadal.org/comunicados/Cuba-y-su-DecretoLey-370.pdf

Cuba, C. d. (15 de noviembre de 2012). Decreto-Ley 305. De las cooperativas no agropecuarias. Gaceta Oficial de la República de Cuba. La Habana, Cuba.

Cuba, c. d. (30 de diciembre de 2017). Decreto-Ley 352. Sobre la adquisición de la ciudadanía cubana por nacimiento de los nacidos en el extranjero de padre o madre cubanos. Gaceta Oficial de la República de Cuba. La Habana, Cuba.

Cuba, C. d. (4 de julio de 2019). Decreto-Ley 370, sobre la Informatización de la Sociedad en Cuba. Gaceta Oficial de la República de Cuba. La Habana, Cuba.

Cuba, P. C. (1975 a Habana). Resoluciones y Tesis del Primer Congreso del Partido Comunista de Cuba sobre la Constitución y la Ley de Tránsito Constitucional. La Habana, Cuba: Editora Política. Obtenido de Granma.

Cubadebate. (21 de diciembre de 2018). Cubadebate Recuperado de: http:// www.cubadebate.cu/especiales/2018/12/21/cuales-son-los-principales-cambios-en-el-proyecto-de-constitucion/\#.XxgwYZ5Khyw

Cubadebate. (1 de marzo de 2019). Cubadebate. Recuperado de Cubadebate: http://www.cubadebate.cu/noticias/2019/03/01/comision-electoral-nacional-fija-cifras-definitivas-90-15-de-electores-votaron-en-referendo-constitucional/\#.X6frCNBKhyw

EFE. (16 de febrero de 2019). Cuba inicia referendo constitucional con el voto de diplomáticos en el exterior. El Diario.es. Recuperado de https://www.eldiario. es/politica/cuba-referendo-constitucional-diplomaticos-exterior_1_1694188. html

Europea, U. (2017). Informe 12816/17 sobre la situación de los Derechos Humanos en Cuba.

Exteriores, M. d. (12 de marzo de 2018). Ministerio de Relaciones Exteriores. Recuperado de Ministerio de Relaciones Exteriores: http://misiones.minrex.gob.cu/ es/articulo/masiva-participacion-de-cubanos-en-comicios-generales

Exteriores, M. d. (27 de agosto de 2018). Nación y Emigración. Recuperado de Nación y Emigración: http://www.nacionyemigracion.cu/content/c-mo-participar-en-el-proceso-de-consulta-de-la-constituci-n-desde-el-exterior

Gargarella, R. (2014). La sala de máquinas de las constituciones latinoamericanas. Buenos Aires, Argentina: Katz.

Humanos, C. I. (2019). Informe Anual. Recuperado de http://www.oas.org/es/ cidh/expresion/informes/ESPIA2019.pdf

Humanos, C. I. (2020). Comunicado de Prensa R78/20. Recuperado de http:// www.oas.org/es/cidh/expresion/showarticle.asp? $\operatorname{art} \mid \mathrm{D}=1173 \& \mathrm{ID}=2$

Jimenez Enoa, A. (17 de agosto de 2017). El Estornudo. Recuperado de Recuperado de: https://www.revistaelestornudo.com/cuba-ejercito-troles/

Lachapelle, J. L. (2020). Social Revolution and Authoritarian Durability. World Politics, 1-44. 
Orrio, M. D. (20 de diciembre de 2013). Rebelión. Recuperado de Rebelión: https://rebelion.org/cuba-temas-y-como-va-el-poder-popular/

Padrón Cuento, C. (16 de junio de 2020). 14ymedio. Recuperado de 14ymedio: https://www.14ymedio.com/cuba/cubanos-multados-ley-mordaza_0_2894710505.html

Popular, A. N. (29 de octubre de 1992). Ley Electoral. Gaceta Oficial de la República de Cuba. La Habana, Cuba.

Popular, A. N. (10 de abril de 2019). Constitución de la República de Cuba. Gaceta Oficial de la República de Cuba. La Habana, Cuba.

Popular, A. N. (19 de agosto de 2019). Ley 127: Electoral. Gaceta Oficial de la República de Cuba. La Habana, Cuba.

Popular, A. N. (16 de enero de 2020). Ley 131: de la Organización y Funcionamiento de la Asamblea Nacional del Poder Popular y el Consejo de Estado. Gaceta Oficial de la República de Cuba. La Habana, Cuba.

Prieto, M. (2005). Reflexiones en torno al carácter normativo de la constitución. En M. \&. Prieto, Temas de Derecho Constitucional Cubano. La Habana, Cuba: Felix Varela.

Prieto, M. (2008). En pos de la aplicabilidad directa de la constitución cubana de 1976 (un breve comentario). IUS. Revista del Instituto de Ciencias Jurídicas de Puebla, 193-205.

Rodríguez Parrilla, B. (28 de octubre de 2017). Cubadebate. Recuperado de Cubadebate: http://www.cubadebate.cu/noticias/2017/10/28/cuba-anuncia-nuevas-medidas-en-vinculos-con-su-emigracion/\#.X2yfuWhKhyw

Rodríguez, C. R. (1983). Entrevista a Teresa Gurza. En C. R. Rodríguez, Letra con filo. La Habana, Cuba: Ciencias Sociales.

Service, I. P. (7 de junio de 2018). Inter Press Service. Recuperado de Inter Press Service: https://www.ipscuba.net/genero/carta-de-lideres-religiosos-cubanos-aviva-debate-sobre-matrimonio-igualitario/

Shahbaz, A. (2019). Freedom House. Recuperado de Freedom House: https://freedomhouse.org/report/freedom-net/2019/crisis-social-media

Toque, E. (18 de agosto de 2018). YouTube. Recuperado de: https://www.youtube.com/watch? $\mathrm{V}=\mathrm{wmSJ}$ ob3Hwi4\&feature $=$ emb_title

Toque, E. (19 de diciembre de 2019). El Toque. Recuperado de El Toque: https://eltoque.com/107-normas-juridicas-que-se-aprobaran-en-cuba-hasta-2028-cronograma-legislativo/

Toque, E. (26 de diciembre de 2019). El Toque. Recuperado de El Toque: https://eltoque.com/107-normas-juridicas-que-se-aprobaran-en-cuba-hasta-2028-cronograma-legislativo/

Unidas, A. G. (24 de octubre de 1970). Resolución 2626. New York, Estados Unidos.

Unidas, R. E. (2019). Internal Communication, AL CUB 5/2019. Recuperado de https://spcommreports.ohchr.org/TMResultsBase/DownLoadPublicCommunicationfile? gld $=24788$

Welp, Y. (2020). Deliberation in the process of constitutional reform: Cuba comparative context. Foro Europa-Cuba. 